\title{
Análise faunística de Calliphoridae (Diptera) da Reserva Biológica do Tinguá, Nova Iguaçu, Rio de Janeiro
}

\author{
Adriana Cristina P. Ferraz¹, Bárbara de Q. Gadelha² \& Valéria M. Aguiar-Coelho²
}

${ }^{1}$ Pós-graduação Biologia Animal, Univ. Federal Rural do Rio de Janeiro. Rod. BR 465, km 7, 23890-000 Seropédica-RJ, Brasil. adrianapedroso7@yahoo.com.br

${ }^{2}$ Depto. Microbiologia e Parasitologia, Univ. Federal do Estado do Rio de Janeiro. Rua Frei Caneca, 94, Centro, 20211-040 Rio de Janeiro-RJ Brasil.brgsqn@yahoo.com.br, valeria@unirio.br.

\begin{abstract}
Analysis of Calliphoridae (Diptera) fauna in Reserva Biológica do Tinguá, Nova Iguaçu, Rio de Janeiro. The present study investigates the calliphorid fauna in the Biological Reserve of Tinguá using faunistic indices. Monthly samplings were carried out using traps containing sardines that were exposed for 48 hours at the sites: A, at the border of the forest; B, 1,000 m towards the interior of the forest; and C, $500 \mathrm{~m}$ towards the interior of the forest. The objective was to study the Calliphoridae fauna in forest environments using faunal indices. During the course of the experiment, 26 calliphorid species were collected. The species Singletons, Doubletons, Uniques, Duplicates, intermediate and common were defined. Richness and richness estimation (estimators Chao 1 and 2, Jackknife 1 and 2, Ace, Ice and Bootstrap), diversity (Shannon-Wiener index), evenness (Pielou's index), and similarity of the sites (using the Sorensen coefficient and the Southwood percent similarity) were calculated. Sites A and B had the same number of species of Calliphoridae (23), representing a greater richness than site C (16). The sites differed only in their rare, intermediate and common species composition. The greatest number of intermediate species was found at A. At B, the numbers of rare, intermediate and common species were similar. At $\mathrm{C}$, there were fewer rare species than intermediate and common ones. Jackknife 2 at site B produced a higher value, however, indicating that it was possible to collect five other species in the reserve and three more species at A and C. Diversity was greatest at B, but evenness was similar at the sites. B and C showed a greater level of similarity in species composition (dendogram); L. nigripes and Mesembrinella bellardiana, which are the most important species at these two sites, appeared grouped together. Chrysomya albiceps, Chrysomya megacephala and Hemilucilia semidiaphana, the main species at Site A, also appear together.
\end{abstract}

KEYWORDS. Blow fly; diversity; evenness; variety.

RESUMO. Análise faunística de Calliphoridae (Diptera) da Reserva Biológica do Tinguá, Nova Iguaçu, Rio de Janeiro. O presente estudo investiga a fauna de califorídeos na Reserva Biológica do Tinguá usando como meios os índices faunísticos. Realizaram-se coletas mensais com armadilhas contendo sardinha expostas por 48 horas, nos pontos: A- borda da mata; B- 1000 metros para interior da mata; C- 500 metros para interior da mata. Objetivou-se estudar a entomofauna califorídica em ambiente florestal através de índices faunísticos. Foram coletadas 26 espécies de califorídeos ao longo do experimento. Definiram-se espécies Singletons, Doubletons, Uniques e Duplicates, intermediárias e comuns; calcularamse riqueza e projeção da riqueza (estimadores Chao 1 e 2, Jackknife 1 e 2, Ace, Ice e Bootstrap); diversidade (Índice Shannon-Wiener); equidade (Índice Pielou) e similaridade dos pontos (quociente Sorensen e porcentagem de similaridade Southwood). Os pontos A e B mostraram o mesmo número de espécies de califorídeos (23), maior que C (16), diferindo apenas nas espécies raras, intermediárias e comuns. Em A foi registrado grande número de espécies consideradas intermediárias. Em B o número de espécies raras, intermediárias e comuns foi similar. Em $\mathrm{C}$, o número de espécies raras foi menor que de intermediárias e comuns. Jackknife 2 no ponto B gerou a maior riqueza, indicando possibilidade de serem coletadas mais cinco espécies na reserva além das coletadas neste estudo, e em A e C mais três espécies. Ace e Bootstrap apresentaram-se seguros para estimativa de riqueza de Calliphoridae. A diversidade foi maior em B. A equidade foi semelhante nos pontos. B e C foram mais semelhantes em relação às espécies (dendograma): Laneela nigripes e Mesembrinella bellardiana, principais destes dois pontos aparecem agrupadas; Chrysomya albiceps, Chrysomya megacephala e Hemilucilia semidiaphana, principais espécies em A aparecem unidas.

PALAVRAS-CHAVE. Califorídeos; diversidade; equidade; riqueza.

A biodiversidade é regulada por vários fatores, em várias escalas, sendo esses fatores locais ou regionais (Moreno 2001). Em uma mesma área geográfica, a diversidade reflete a coexistência entre os organismos, que interagem através da competição por utilizarem as mesmas fontes ou o mesmo ambiente (Whittaker 1967). Em ambientes em equilíbrio competitivo, a diversidade total de espécies apresenta-se normalmente baixa e as espécies são suficientemente diferentes para coexistirem, evitando a competição por fontes críticas (Huston 1995).

A perda de biodiversidade pela ação humana é um dos maiores motivos para estabelecimento de áreas de proteção ambiental (Moreno 2001), e tem sido objeto de amplos debates (Magurran 1988).

Muitos projetos conservacionistas utilizam análises de riqueza e diversidade para demonstrar suas relevâncias (Dias 
2004). Modelos matemáticos de estudo proporcionam uma idéia da estrutura e funcionamento da comunidade e daí então se realizam métodos mais complexos para aumentar conhecimento de grupos animais (Southwood 1995) e estabelecer as melhores estratégias.

Atualmente sabe-se que a diversidade de organismos é maior nos trópicos, principalmente quando analisamos a classe dos insetos (Primack \& Rodrigues 2001). Os insetos podem se tornar indicadores ecológicos em ambientes que estejam sofrendo impacto (Margalef 1951) devido sua diversidade e capacidade de produzir diversas gerações em curto espaço de tempo (Holloway et al 1987), além de ser o grupo taxonômico que representa maioria da biodiversidade terrestre (Moreno 2001).

Alternativas rápidas e confiáveis para medição aproximada da diversidade biológica, sem dados exaustivos se fazem necessárias para respondermos rápido aos processos de perda dessa biodiversidade (Moreno 2001).

Os califorídeos têm um importante papel na ciclagem de nutrientes no ambiente terrestre, uma vez que suas larvas atuam como decompositoras da matéria orgânica. Esta característica pode ser utilizada para estudos de entomologia forense (Moura et al. 1997) e acelerando processos de cicatrização na terapia larval (Neves 2005). Podem atuar também como polinizadores (Silva et al. 2001), veiculadores de patógenos (Greenberg 1973) e agentes causadores de miíases em vertebrados vivos (Guimarães et al. 1983). Esses dípteros possuem grande capacidade de adaptação ao ambiente modificado pelo homem, sendo algumas espécies classificadas como sinantrópicas (Furusawa \& Cassino 2006). Ao avaliar a distribuição das espécies, pode-se observar os níveis de adaptação às modificações ambientais, verificando que diferentes espécies se distribuem em distintos locais.

O presente estudo tem como objetivo comparar três pontos da Reserva Biológica do Tinguá (da borda ao interior da mata) através de análises faunísticas (frequiência, riqueza real e estimada, diversidade e equitabilidade) das espécies de califorídeos capturadas ao longo do período de estudo.

\section{MATERIALE MÉTODOS}

A Reserva Biológica do Tinguá-RJ (REBIO - Tinguá), reserva de 24.903,00 (ha) (Ibama 2006), é a maior concentração de Mata Atlântica Primária do Rio de Janeiro e está localizada na Serra do Mar entre os municípios de Nova Iguaçu, Duque de Caxias, Petrópolis, Miguel Pereira e Vassouras (Silva 2001).

Para captura dos insetos foram utilizadas seis armadilhas de fabricação caseira, tendo a base preta com orifícios para a entrada dos dípteros muscóides, onde se encontrava a isca atrativa. Acoplada a essa parte encontra-se um recipiente plástico transparente para a entrada de luz (Mello et al 2007). Foi utilizado como atrativo para os insetos $400 \mathrm{~g}$ de sardinha congelada em freezer e descongelada 24 horas em geladeira antes da exposição a campo. As coletas dos dípteros foram mensais, realizadas de junho de 2006 a maio de 2007.

As armadilhas foram colocadas em três pontos pré-

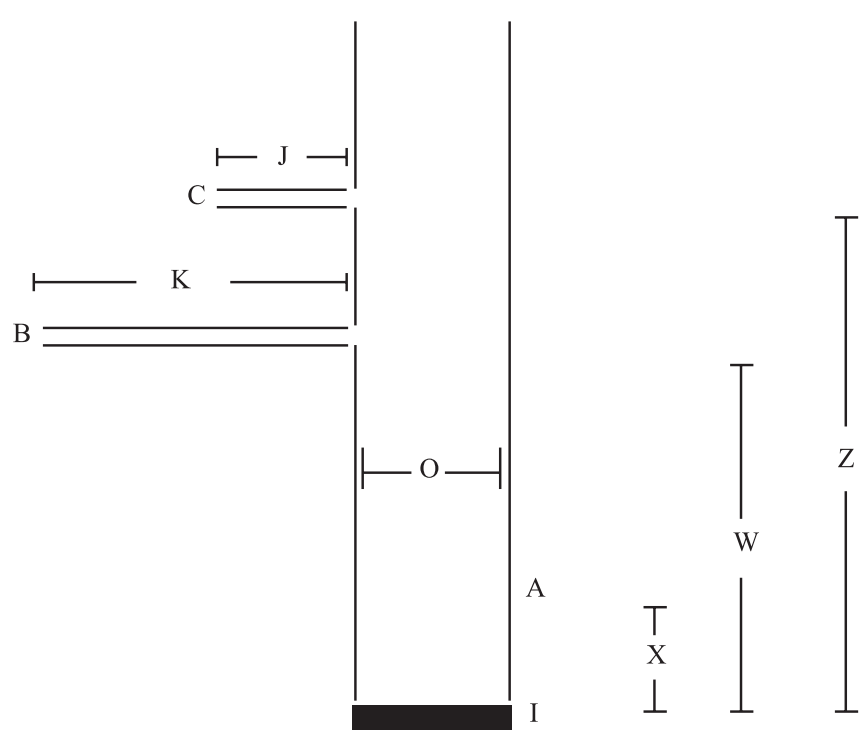

Fig. 1. Esquema da localização dos pontos de coleta (A, B e C) de dípteros da família Calliphoridae na Reserva Biológica do Tinguá, RJ. $\mathrm{I}=$ portão de acesso da reserva, $\mathrm{O}=$ Orbel $1, \mathrm{X}=500$ metros, $\mathrm{W}=1200$ metros, $Z=1700$ metros, $K=1000$ metros, $\mathrm{J}=500$ metros.

definidos registrados com GPS (Global Positioning System) próximos à Sede Administrativa (S 22 $58.559^{\prime}$, W 4343.809'), localizada em Nova Iguaçu, variando da borda ao interior da mata, buscando evidenciar a relação entre o efeito de borda sobre a população de califorídeos. O primeiro (Ponto A - S $22^{\circ} 58.788^{\prime} \mathrm{W} 43^{\circ} 43.459^{\prime}$ ) se localizou na borda da mata, próximo 500 metros da entrada da Reserva. O segundo (Ponto B - S $22^{\circ} 58.523^{\prime} \mathrm{W} 43^{\circ} 44.540^{\prime}$ ) estava localizado a $1200 \mathrm{~m}$ da entrada da Reserva, seguindo-se pela extinta Estrada do Comércio (ou Estrada do Ouro) e a $1000 \mathrm{~m}$ para o interior da mata no sentido perpendicular a esta estrada. O terceiro (Ponto C - S $22^{\circ} 58.350^{\prime} \mathrm{W} 43^{\circ} 44.678^{\prime}$ ) estava localizado a $1700 \mathrm{~m}$ da entrada da Reserva, seguindo-se por esta estrada, a $500 \mathrm{~m}$ da borda (Figura 1). As espécies vegetais mais representativas em cada ponto foram: cactos (Cactaceae), jaqueiras (Artocarpus heterophyllus) e bromélias (Bromeliaceae) no ponto $\mathrm{A}$, bambus (Bambusoideae) no ponto B e bromélias (Bromeliaceae) no ponto C.

Cada ponto recebeu duas armadilhas pretas para não ocorrer influência pela cor das armadilhas (Ferraz \& AguiarCoelho 2008), afastadas cinco metros entre si. A altura das armadilhas foi de $1,5 \mathrm{~m}$ do solo.

Após 48 horas de exposição das armadilhas a campo, estas foram retiradas e os insetos foram sacrificados com a utilização de algodão umedecido com éter, para então serem transferidos para frascos com álcool 70\%. No Laboratório de Estudo de Dípteros (LED) da Universidade Federal do Estado do Rio de Janeiro (UNIRIO), os insetos foram retirados do álcool com auxílio de uma peneira, transferidos para papel absorvente e secos com luz incidente. Estes então sofreram processo de alfinetagem direta e os califorídeos foram identificados utilizando microscópio estereoscópico pela chave de identificação de Mello (2003). Os exemplares testemunhas 
foram depositados na Coleção Entomológica do Laboratório de Estudo de Dípteros (LED) da UNIRIO.

Após a análise dos resultados, realizou-se uma análise faunística em que se considerou cada ponto de coleta como uma comunidade diferente.

1. Freqüência de espécies na comunidade:

Foram definidas espécies raras aquelas que tiveram um ou dois indivíduos coletados por localidade, chamados respectivamente de Singletons e Doubletons. As espécies encontradas somente em uma amostra foram consideradas Uniques e aquelas encontradas em apenas duas amostras foram chamadas Duplicates), intermediárias as espécies com três a 51 indivíduos, e comuns as com 52 ou mais indivíduos (Krüger 2006).

2. Estimativas não paramétricas de riqueza de espécies:

Segundo Colwell (2004), os estimadores que se baseiam na riqueza das espécies raras fazem uso das quatro variáveis: singletons, doubletons, uniques e duplicates.

O estimador Chaol é baseado na abundância e utiliza a relação entre o número de Singletons e Doubletons (Colwell 2004). A fórmula para Chao 1 (Chao 1984, 1987) é : Chao $1=$ $\mathrm{S}_{\text {obs }}+\left(\mathrm{a}^{2} / 2 \mathrm{~b}\right)$, onde $S_{o b s}$ é o número de espécies observado nas amostras, $a$ é o número de espécies representadas por apenas um espécime, e $b$ é o número de espécies representado por exatamente dois espécimes.

Para Chao 2 (Chao 1984, 1987) é : Chao $2=\mathrm{S}_{\mathrm{obs}}+\left(\mathrm{L}^{2} / 2 \mathrm{M}\right)$, onde $S_{o b s}$ é o número de espécies observado nas amostras, $L$ é o número de espécies representadas em somente uma amostra, e $m$ é o número de espécies representado em somente duas amostras.

A fórmula para Jackknife de primeira ordem (Jack 1): Jack $l=\mathrm{S}_{\mathrm{obs}}+\mathrm{L}(\mathrm{n}-1 / \mathrm{n})$, e segunda ordem (Jack 2): Jack $2=\mathrm{S}_{\mathrm{obs}}+[\mathrm{L}$ $\left.(2 \mathrm{n}-3) / \mathrm{n}-\mathrm{M}(\mathrm{n}-2)^{2} / \mathrm{n}(\mathrm{n}-1)\right]$, onde $\mathrm{S}_{\mathrm{obs}}$ é o número de espécies observado nas amostras, $\mathrm{M}=$ número de espécies que ocorrem em exatamente duas amostras, L é o número de espécies representadas em somente uma amostra e n é o número de amostras (Palmer 1991).

O estimador Ace é baseado no conceito de abundância e utiliza para as estimativas de riqueza espécies com dez ou menos indivíduos por amostra, que não são singletons (Lee \& Chao 1994). Sua fórmula é: $\mathrm{S}_{\text {ace }}=\mathrm{S}_{\text {abund }}+\left(\mathrm{S}_{\mathrm{rare}} / \mathrm{C}_{\mathrm{ace}}\right)+\left(\mathrm{F}_{1} / \mathrm{C}_{\text {ace }}\right)$ $\gamma_{\text {ace }}^{2}$, onde: $\mathrm{S}_{\mathrm{obs}}=$ número total de espécies observadas em todas as amostras agrupadas $\left(\mathrm{S}_{\text {obs }}=\mathrm{S}_{\text {rare }}+\mathrm{S}_{\text {abund }}\right), \mathrm{S}_{\text {rare }=}$ número de espécies raras (cada com 10 ou menos indivíduos) quando todas as amostras estão agrupadas, $\mathrm{S}_{\text {abund }}=$ número de espécies abundantes (cada com 10 ou mais indivíduos) quando todas as amostras estão agrupadas, $\mathrm{C}_{\text {ace }}=$ Estimador da cobertura da abundância da amostra $\left(C_{\text {ace }}=1-F_{I} / N_{\text {rare }}\right), \mathrm{F}_{\mathrm{i}}=$ número de espécies que tem exatamente $i$ indivíduos quando todas as amostras estão agrupadas ( $F_{1}$ é a frequência de singletons, $F_{2}$ é a freqüência de doubletons), $\mathrm{N}_{\text {rare }}=$ número total de indivíduos em espécies raras, $\gamma_{\text {ace }}^{2}$ coeficiente estimado de variação de Fi para espécies raras $\left(\gamma_{\text {ace }}^{2}=\max \left[\left(S_{\text {rare }} / C_{\text {ace }}\right) * i(i-1) F i /\right.\right.$ $\left.\left(N_{\text {rare })}\left(N_{\text {rare }}-1\right)-1,0\right]\right)$.

$\mathrm{O}$ estimador Ice é baseado em incidência, utilizando espécies encontradas em 10 ou menos amostras que não são

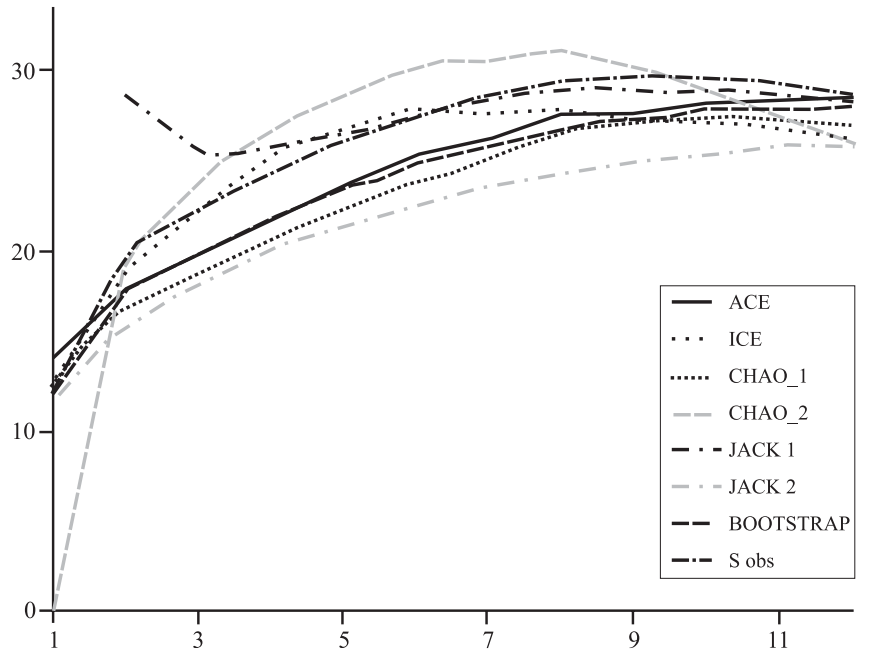

Fig. 2. Estimativas de riqueza de espécies de califorídeos na Reserva Biológica do Tinguá, RJ. $\mathrm{S}_{\mathrm{obs}}=$ espécies observadas.

uniques (Lee \& Chao 1994). A fórmula para seu cálculo é: $\mathrm{S}_{I c e}$ $=\mathrm{S}_{\text {freq }}+\left(\mathrm{S}_{\text {infr }} / \mathrm{C}_{\text {Ice }}\right)+\left(\mathrm{Q}_{\mathrm{j}} / \mathrm{C}_{\text {Ice }}\right) \gamma_{\text {Ice }}^{2}$ onde: $\mathrm{S}_{\text {obs }}=$ número total de espécies observadas em todas as amostras agrupadas $\left(\mathrm{S}_{\mathrm{obs}}=\right.$ $\mathrm{S}_{\text {infr }}+\mathrm{S}_{\text {freq }}$ ), $\mathrm{S}_{\text {freq }}=$ = número de espécies freqüentes (cada uma encontrada em mais que 10 amostras), $\mathrm{S}_{\text {infr }}=$ número de espécies infreqüentes (uma encontrada em menos de 10 amostras), $\mathrm{C}_{I c e}=$ Estimador da cobertura da incidência da $\operatorname{amostra}\left(C_{I c e}=1-Q_{j} / N_{i n f r}\right), \mathrm{Q}_{\mathrm{j}}=$ número de espécies que ocorre em exatamente j amostras $(Q 1$ é a frequiência de uniques, Q2 é a freqüência de duplicates), $\mathrm{N}_{\mathrm{inf}}=$ número total de ocorrências de espécies infrequentes, $\gamma_{\text {Ice }}^{2}=$ coeficiente estimado de variação de $Q i$ para espécies infreqüentes $\left(\gamma_{\text {Ice }}^{2}=\right.$ $\left.\max \left[\left(S_{i n f r} / C_{\text {Ice }}\right)\left(m_{i n f r} / m_{i n f r-1}\right) * \sum j(j-1) Q_{j} /\left(N_{i n f r}\right)^{2}-1,0\right]\right) . \mathrm{S}_{\text {inf } \mathrm{r}}=$ número de espécies infrequentes (cada uma encontrada em 10 ou menos amostras), $\mathrm{m}_{\text {inf } \mathrm{r}}=$ número de amostras que tem pelo menos uma espécie infreqüente.

Bootstrap é um estimador baseado também na incidência de espécies (Smith \& Van Belle 1984): $\mathrm{S}_{\text {boot }}=\mathrm{S}_{\text {obs }}+\sum\left(1-\mathrm{p}_{\mathrm{k}}\right)^{\mathrm{m}}$, em que: $\mathrm{S}_{\text {obs }}=$ número total de espécies observadas em todas as amostras agrupadas, $\mathrm{p}_{\mathrm{k}}=$ proporção de amostras que contém a espécie $k, \mathrm{~m}=$ número total de amostras.

A projeção da riqueza foi obtida por meio dos estimadores de riqueza Chao 1, Chao 2, Jackknife de primeira e segunda ordem, Ace, Ice e Bootstrap, calculados através do programa EstimateS Win 8.0 (Colwell 2006). Os dados foram aleatorizados 100 vezes. Estes métodos de análise foram escolhidos por serem destinados a analisar dados com diferentes distribuições de abundância (Santos 2003).

3. Diversidade e equidade:

Os índices de diversidade de espécies foram calculados pela função de Shannon-Wiener: $\mathrm{H}^{\prime}=-\sum(f i) \log (f i)$, onde $f i$ é a proporção dos indivíduos pertencentes a n-ésima espécie e $l n$ é o logaritmo neperiano (Pielou 1975). Para provar a hipótese nula de que as amostras (pontos A, B e C, medidos com Índice de Shannon) são iguais, procedeu-se conforme Hutcheson (1970) (apud Zar 1999). Para cada amostra se calculou o índice 
de diversidade ponderado (Hp) em função da frequiência de cada espécie: $H p=(N \log N)$ “ $\left(\sum f i \log f i\right) / N$, onde $f i=$ freqüência (número de indivíduos) registrada para a espécie $i$, e a variância do índice de diversidade ponderado: $\operatorname{var}=\left[\sum f i \log ^{2} f i\right.$ " $\left(\sum f i \log \right.$ $\left.f i)^{2}\right] / N / N^{2}$. Calculou-se a diferença das variâncias de ambas as amostras: $D_{\text {var }}=$ " (var $\left.1+\operatorname{var} 2\right)$. Obteve-se o valor de $t: t=$ $H p_{1}-H p_{2} / D_{\text {var }}$ Calculou-se o grau de liberdade associado com o valor de $t$ : $g . l .=\left(\operatorname{var}_{1}+\operatorname{var}_{2}\right)^{2} /\left(\operatorname{var}_{1}^{2} / N_{1}\right)+\left(\operatorname{var}_{2}^{2} / N_{2}\right)$. Comparou-se o $t$ calculado com $t$ tabelado.

Os índices de eqüidade (J') foram calculados segundo Pielou (1966): J' = H'/H $\mathrm{H}_{\max }$, onde H' é o índice de ShannonWiener e $\mathrm{H}_{\max }$ é o logaritmo neperiano (ln) do número total de espécies na amostra. Este índice varia de 0 a 1 e avalia a uniformidade de captura das espécies ao longo da amostragem (Magurran 1988).

4. Similaridade entre as áreas:

Para a medida de similaridade entre as áreas estudadas, no que diz respeito à composição de espécies, calculou-se o quociente de similaridade proposto por Sorensen (1948): Q.S. $=2 \mathrm{c} / \mathrm{a}+\mathrm{b}$, onde $a$ e $b$ são os números de espécies que ocorrem nos habitats $X$ e $Y$; $c$ é o número de espécies presentes em ambos os habitats. Essa análise é baseada na presença/ ausência das espécies.

A fim de se observar a semelhança entre as comunidades das áreas estudadas, não só em termos de composição de espécies, mas também em relação ao número de indivíduos coletados, foi utilizado o cálculo da porcentagem de similaridade (Southwood 1978): PS $=\sum$ da menor percentagem para cada espécie quando comparadas as duas áreas.

5. Análise de Agrupamento:

As espécies presentes nos três pontos foram comparadas através da análise de agrupamento quantitativa para verificar se as espécies estão agrupadas em função do tipo de habitat (Zar 1999) e ainda foi construído outro dendograma comparando a similaridade entre os locais baseando-se na lista de espécies. Ambos utilizando a distância euclidiana e realizados com auxílio do programa Statistica edição 1999.

\section{RESULTADOS}

Foram coletados 8516 espécimes de califorídeos distribuídos em 26 espécies em 11 gêneros. No ponto A foram coletadas 23 espécies, sendo Chrysomya albiceps (Wiedemann, 1819) a espécie dominante representando cerca de $29,85 \%$ dos califorídeos coletados neste ponto. No ponto B, foram coletadas também 23 espécies, sendo 29,69\% pertencentes à espécie Mesembrinella bellardiana (Aldrich, 1922). Já no ponto C, foram coletadas 16 espécies e a dominância ocorreu por conta da espécie Laneela nigripes (Guimarães, 1977) com 51,13\% dos espécimes identificados (Tabela I).

Os pontos A e $\mathrm{B}$ apresentaram maior proporção de espécies comuns, enquanto B apresentou a maior porcentagem de espécies raras, e A a maior proporção de espécies intermediárias. Nos pontos A e C, ocorreu um maior número de espécies intermediárias enquanto no B o número de espécies

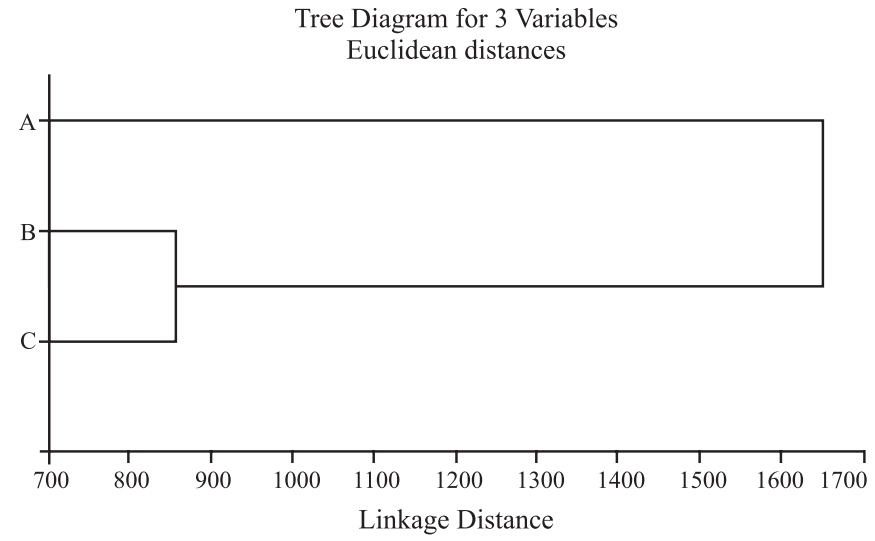

Fig. 3. Dendograma comparando a similaridade entre os pontos de coleta na Reserva Biológica do Tinguá baseando-se na lista de espécies.

raras, intermediárias e comuns foi muito semelhante (Tabela II).

Durante todo o período de coleta, do total de espécies coletadas, três tiveram um único indivíduo (Singletons), dois tiveram dois indivíduos (Doubletons), três foram encontradas somente em uma amostra (Uniques) e sete foram encontradas em duas amostras (Duplicates). Em relação aos pontos, A e C apresentaram um número distribuído de Uniques, Duplicates, Singletons e Doubletons (4/4/3/1, no ponto A e 3/2/2/1, no ponto $\mathrm{C}$, respectivamente). Já o ponto $\mathrm{B}$ teve o maior número de espécies Singletons (7), e ainda 5 Duplicates, 4 Singletons e 4 Doubletons .

A estimativa de riqueza total gerada pelo estimador Jackknife 2 foi a mais baixa de todas com aproximadamente 26 espécies e a Jackknife 1 a mais alta com aproximadamente 29 espécies. Os estimadores Ace e Ice encontraram valores muito próximos, aproximadamente 28 espécies (Tabela III).

No ponto A, as estimativas de riqueza variaram de 24 (estimador Chao 2) a 27 espécies (Jackknife 2), no ponto B de 24 (Chao 1) a 31 espécies (Jackknife 2), e no ponto C de 16 (Chao 1) a 19 espécies (Jackknife 2). Segundo o estimador Chao 1, os pontos A e B seriam muito semelhantes. Em todos os estimadores, o ponto $\mathrm{C}$ foi o que apresentou a menor riqueza. As curvas de acumulação de espécies dos estimadores estão descritas na Figura 2.

O índice de diversidade $\mathrm{H}^{\prime}=2,01$, obtido para o ponto B, foi maior que aquele encontrado para o $\mathrm{A}, \mathrm{H}^{\prime}=1,87$, e C H' = 1,61. A hipótese nula de que as amostras (pontos A, B e C, medidos com Índice de Shannon) são iguais foi recusada (conforme Hutcheson (1970), citado por Zar (1999) e, portanto, os pontos A, B e C são diferentes. Os índices de equidade foram: $\mathrm{J}^{\prime}=0,23$, encontrado para o ponto $\mathrm{A}, \mathrm{J}{ }^{\prime}=0,25$ obtido para o ponto $\mathrm{B}$, e J' $=0,22$ para o ponto $\mathrm{C}$.

Todas as espécies amostradas no ponto $\mathrm{C}$ foram encontradas no ponto $\mathrm{B}$, proporcionando um coeficiente de similaridade alto (Q.S. $=0,69)$ entre as áreas. Porém, a maior similaridade foi entre os pontos A e B (Q.S. $=0,87$ ) e a menor foi ente A e C (Q.S. = 0,61).

A porcentagem de similaridade entre as áreas $\mathrm{A}$ e $\mathrm{C}$ (PS = 
$37,27 \%$ ) foi relativamente baixa, enquanto as porcentagens encontradas entre A e B ( $\mathrm{PS}=51,95 \%)$ e B e C (PS = 63,26\%), foram mais semelhantes.

A análise quantitativa (Distância Euclidiana) agrupou com maior similaridade as espécies que ocuparam os pontos $\mathrm{B}$ e C (Figura 3). A similaridade entre as cinco espécies mais coletadas na Reserva Biológica do Tinguá durante o período do estudo em função do habitat está demonstrada na Figura 4.

\section{DISCUSSÃO}

A riqueza de espécies refere-se à abundância numérica de uma determinada área geográfica, região ou comunidade (Rodrigues 2004), ou seja, sua quantidade de espécies. A riqueza e a abundância juntas formam a diversidade (Dias 2004). Segundo Magurran (1988), a riqueza pode ser calculada de diversas formas para um mesmo conjunto de dados, podendo ter métodos que se ajustem melhor do que outros (SilveiraNeto et al. 1976). Ela é vastamente usada pois seu significado

Tabela I. Número de indivíduos (n) e porcentagem (p) de espécies de califorídeos, total de indivíduos coletados $(\mathrm{N})$ e riqueza (S) utilizando armadilhas contendo sardinha em cada ponto de coleta* na Reserva Biológica do Tinguá, junho de 2006 a maio de 2007.

\begin{tabular}{|c|c|c|c|c|c|c|}
\hline \multirow[t]{2}{*}{ Espécies } & \multicolumn{2}{|r|}{ A } & \multicolumn{2}{|c|}{ B } & \multicolumn{2}{|c|}{$\mathrm{C}$} \\
\hline & $\mathrm{n}$ & $\mathrm{p}$ & $\mathrm{n}$ & $\mathrm{p}$ & $\mathrm{n}$ & $\mathrm{p}$ \\
\hline Calliphora vicina & 0 & 0.00 & 1 & 0.03 & 0 & 0.00 \\
\hline Chloroprocta idioidea & 18 & 0.46 & 3 & 0.10 & 8 & 0.48 \\
\hline Chrysomya albiceps & 1161 & 29.85 & 256 & 8.70 & 67 & 3.98 \\
\hline Chrysomya megacephala & 729 & 18.74 & 49 & 1.66 & 40 & 2.38 \\
\hline Chrysomya putoria & 10 & 0.26 & 2 & 0.07 & 0 & 0.00 \\
\hline Cochliomyia hominivorax & 7 & 0.18 & 2 & 0.07 & 1 & 0.06 \\
\hline Cochliomyia macellaria & 59 & 1.52 & 13 & 0.44 & 0 & 0.00 \\
\hline Eumesembrinella besnoiti & 3 & 0.08 & 1 & 0.03 & 0 & 0.00 \\
\hline Eumesembrinella pauciseta & 13 & 0.33 & 52 & 1.77 & 10 & 0.59 \\
\hline Eumesembrinella & & & & & & \\
\hline quadrilineata & 0 & 0.00 & 2 & 0.07 & 4 & 0.24 \\
\hline Eumesembrinella randa & 0 & 0.00 & 2 & 0.07 & 1 & 0.06 \\
\hline Hemilucilia segmentaria & 147 & 3.78 & 122 & 4.14 & 29 & 1.72 \\
\hline Hemilucilia semidiaphana & 1045 & 26.86 & 572 & 19.43 & 195 & 11.59 \\
\hline Huascaromusca aeneiventris & 8 & 0.21 & 24 & 0.82 & 0 & 0.00 \\
\hline Huascaromusca purpurata & 1 & 0.03 & 1 & 0.03 & 0 & 0.00 \\
\hline Laneela nigripes & 302 & 7.76 & 479 & 16.27 & 860 & 51.13 \\
\hline Mesembrinella bellardiana & 214 & 5.50 & 874 & 29.69 & 293 & 17.42 \\
\hline Mesembrinella bicolor & 10 & 0.26 & 17 & 0.58 & 11 & 0.65 \\
\hline Mesembrinella semihyalina & 51 & 1.31 & 109 & 3.70 & 70 & 4.16 \\
\hline Paralucilia borgmeieri & 2 & 0.05 & 0 & 0.00 & 0 & 0.00 \\
\hline Paralucilia fulvinota & 1 & 0.03 & 0 & 0.00 & 0 & 0.00 \\
\hline Paralucilia nigrofacialis & 3 & 0.08 & 1 & 0.03 & 2 & 0.12 \\
\hline Paralucilia paraense & 1 & 0.03 & 0 & 0.00 & 0 & 0.00 \\
\hline Paralucilia pseudo-lyrcea & 34 & 0.87 & 12 & 0.41 & 0 & 0.00 \\
\hline Lucilia eximia & 62 & 1.59 & 331 & 11.24 & 86 & 5.11 \\
\hline Lucilia sericata & 9 & 0.23 & 19 & 0.65 & 5 & 0.30 \\
\hline $\mathrm{N}$ & 3890 & & 2944 & & 1682 & \\
\hline $\mathrm{S}$ & 23 & & 23 & & 16 & \\
\hline
\end{tabular}

*Ponto A- 500 m do portão da Reserva (borda); Ponto B- 1200 m do portão e distando $1000 \mathrm{~m}$ para o interior da mata; Ponto C - $1700 \mathrm{~m}$ do portão e distando $500 \mathrm{~m}$ para o interior da mata.

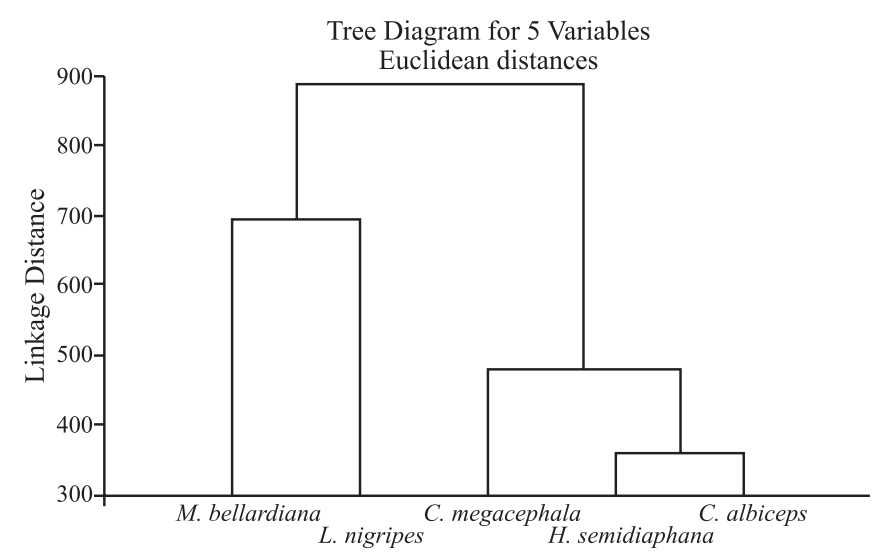

Fig. 4. Dendograma de comparação entre as cinco espécies mais coletadas na Reserva Biológica do Tinguá em função do tipo de habitat.

é amplamente compreendido, existem normalmente muitos dados disponíveis sobre números de espécies, assim como facilmente detectáveis ou quantificáveis (Moreno 2001). Os pontos A e B (mais próximos da entrada da reserva) apresentaram maior riqueza de espécies, que pode ter ocorrido por influxo de espécies não florestais para a floresta.

Apesar da semelhança no número de espécies coletadas entre os pontos A e B existem diferenças entre estes pontos como proximidade da borda, vegetações distintas, entre outros, que podem ter influenciado nas diferentes espécies que ocorreram em cada.

O número de espécies coletadas na Reserva Biológica do Tinguá (26) mostrou-se superior a outros estudos em áreas de preservação como o de Tavares (2003), na Reserva Florestal da Serra do Japi, Jundiaí- SP, que coletou dez espécies de califorídeos em dois anos. Thyssen (2000), em matas naturais da Reserva Municipal de Santa Genebra, Campinas-São Paulo, encontrou sete espécies durante os meses de fevereiro e março de 2007 e agosto e setembro de 2008. Já Ribeiro (2003), em estudo na mata ciliar e cerrado da Reserva Biológica de Mogi Guaçu, São Paulo, coletou quatro espécies em coletas nos meses de fevereiro, julho e agosto de 2000. Estas diferenças podem ser explicadas pelas diferentes metodologias de estudo sendo estes realizados com exposição de carcaças até sua decomposição plena para estudos de entomologia forense, nas quais observou-se que na sucessão faunística os califorídeos são atraídos preferencialmente nas fases de putrefação e putrefação escura (Tavares 2003).

Longino et al. (2002) indicaram que comunidades de insetos em florestas úmidas possuem alto número de espécies raras. No presente estudo, comparando a ocorrência das espécies nos três pontos, o número de espécies raras foi similar às espécies intermediárias e comuns no ponto $\mathrm{B}$, e inferior nos pontos $\mathrm{A} \mathrm{e} \mathrm{C}$, ocorrendo nestes dois pontos um maior número de espécies intermediárias.

Após episódios de fragmentação o número de espécies pode aumentar ou diminuir, ou ainda se manter constante, o que não significa que a fauna não sofreu com a fragmentação 
Tabela II. Número e porcentagem de espécies raras, intermediárias e comuns de califorídeos coletados em armadilhas contendo sardinha em cada ponto de coleta* na Reserva Biológica do Tinguá, junho de 2006 a maio de 2007.

\begin{tabular}{|c|c|c|c|c|c|c|c|c|c|}
\hline & A & $\%$ & Espécies & B & $\%$ & Espécies & $\mathrm{C}$ & $\%$ & Espécies \\
\hline Raras & 4 & 17,39 & $\begin{array}{c}\text { Huascaromusca } \\
\text { purpurata,Paralucilia } \\
\text { fulvinota,Paralucilia } \\
\text { borgmeieri } \mathrm{e} \text { Paralucilia } \\
\text { paraense }\end{array}$ & 8 & 34,78 & $\begin{array}{c}\text { Calliphora } \\
\text { vicina,Chrysomya } \\
\text { putoria,Cochliomyia } \\
\text { hominivorax,Eumesembrinella } \\
\text { besnoiti,Eumesembrinella } \\
\text { quadrilineataEumesembrinella } \\
\text { randa,Huascaromusca } \\
\text { purpurata eParalucilia } \\
\text { nigrofacialis }\end{array}$ & 3 & 18,75 & $\begin{array}{c}\text { Cochliomyia } \\
\text { hominivorax, } \\
\text { Eumesembrinella randa e } \\
\text { Paralucilia nigrofacialis }\end{array}$ \\
\hline Intermediárias & 11 & 47,83 & $\begin{array}{c}\text { Chrysomya putoria, } \\
\text { Chloroprocta idioidea, } \\
\text { Cochliomyia hominivorax, } \\
\text { Eumesembrinella besnoiti, } \\
\text { Eumesembrinella } \\
\text { pauciseta, Huascaromusca } \\
\text { aeneiventris, } \\
\text { Mesembrinella bicolor, } \\
\text { Mesembrinella } \\
\text { semihyalina, Paralucilia } \\
\text { nigrofacialis, Paralucilia } \\
\text { pseudo-lyrcea e Phaenicia } \\
\text { sericata }\end{array}$ & 7 & 30,44 & $\begin{array}{c}\text { Chrysomya megacephala, } \\
\text { Chloroprocta idioidea, } \\
\text { Cochliomyia macellaria, } \\
\text { Huascaromusca } \\
\text { aeneiventris, Mesembrinella } \\
\text { bicolor, Paralucilia pseudo- } \\
\text { lyrcea e Phaenicia sericata }\end{array}$ & 7 & 43,75 & $\begin{array}{c}\text { Chloroprocta idioidea, } \\
\text { Chrysomya } \\
\text { megacephala, } \\
\text { Eumesembrinella } \\
\text { pauciseta, } \\
\text { Eumesembrinella } \\
\text { quadrilineata, } \\
\text { Hemilucilia segmentaria, } \\
\text { Mesembrinella bicolor e } \\
\text { Phaenicia sericata }\end{array}$ \\
\hline Comuns & 8 & 34,78 & $\begin{array}{c}\text { Chrysomya albiceps, } \\
\text { Chrysomya megacephala, } \\
\text { Cochliomyia macellaria, } \\
\text { Hemilucilia segmentaria, } \\
\text { Hemilucilia semidiaphana, } \\
\text { Laneela nigripes, } \\
\text { Mesembrinella bellardiana } \\
\text { e Phaenicia eximia }\end{array}$ & 8 & 34,78 & $\begin{array}{c}\text { Chrysomya albiceps, } \\
\text { Eumesembrinella pauciseta, } \\
\text { Hemilucilia segmentaria, } \\
\text { Hemilucilia semidiaphana, } \\
\text { Laneela nigripes, } \\
\text { Mesembrinella bellardiana, } \\
\text { Mesembrinella semihyalina e } \\
\text { Phaenicia eximia }\end{array}$ & 6 & 37,50 & $\begin{array}{c}\text { Chrysomya albiceps, } \\
\text { Hemilucilia } \\
\text { semidiaphana, Laneela } \\
\text { nigripes, Mesembrinella } \\
\text { bellardiana, } \\
\text { Mesembrinella } \\
\text { semihyalina e Phaenicia } \\
\text { eximia }\end{array}$ \\
\hline
\end{tabular}

*Ponto A- $500 \mathrm{~m}$ do portão da Reserva (borda); Ponto B- $1200 \mathrm{~m}$ do portão e distando $1000 \mathrm{~m}$ para o interior da mata; Ponto C - $1700 \mathrm{~m}$ do portão e distando $500 \mathrm{~m}$ para o interior da mata.

(De Souza et al. 2001). O local de estudo sofreu ação de fragmentação há muito tempo, apesar de o tempo decorrido poder levar a certo tamponamento do efeito de borda, no entanto este corredor desmatado ainda sofre constante manutenção. Como não existem estudos anteriores aos episódios de fragmentação não é possível fazer uma comparação dos efeitos da fragmentação neste local. É necessário, portanto, maior atenção para o estudo de paisagens modificadas pelo homem, situações cada vez mais comuns atualmente (Moreno \& Halffter 2001).

A curva com os valores de espécies observadas $\left(\mathrm{S}_{\text {obs }}\right)$ mostra que no quarto levantamento realizado, já haviam sido coletadas mais de $75 \%$ do total de espécies. O ponto com a maior riqueza esperada foi o B, pelo estimador Jackknife 2, com 31 espécies e Chao 1 resultou nos menores valores, com exceção do ponto A, cujo menor valor foi estimado por Chao 2.

Erwin (1988) em seu trabalho destacou que as curvas de acumulação de espécies esperadas não chegaram a assíntotas e a proporção de espécies raras de insetos foram altas. Porém Longino et al. (2002) apontam que quando as comunidades de insetos possuem alto número de espécies raras, por exemplo, metade de proporção das espécies coletadas, as curvas podem alcançar um platô. No estudo atual, apesar do pequeno número de espécies raras apenas o estimador Jackknife 2 não alcançou um platô.

Todos os estimadores utilizados são algoritmos nãoparamétricos que estimam o número de espécies baseados na quantificação da raridade (Toti et al. 2000) e projetaram estimativas diferentes no presente estudo. O estimador Jackknife 2 teve uma grande ascensão nas primeiras amostras, enquanto que o Ice teve certa queda rapidamente, o que segundo Bragagnolo \& Pinto-da-Rocha (2003) pode sugerir que estes sejam pouco confiáveis quando o número de amostras é pequeno. Entretanto, segundo Colwell \& Coddington (1994) Chao 2 e Jackknife 2 fornecem as estimativas com maior acuidade e menor viés para conjunto de dados com pequeno número de amostras. Um estimador deve alcançar ou aproximar-se da estabilidade com menos amostras do que a curva de acumulação de espécies observadas. Além disso, um estimador não deve apresentarse largamente diferente dos demais (Toti et al. 2000).

Apesar da riqueza nos pontos A e B serem igualmente de 23 espécies, nas estimativas de riqueza, o ponto B apresentou 
Tabela III. Riqueza estimada pelos estimadores Ace, Ice, Chao 1 e 2, Jackknife 1 e 2, e Bootstrap de califorídeos em cada ponto de coleta* na Reserva Biológica do Tinguá.

\begin{tabular}{ccccc}
\hline Estimador & A & B & C & A, B, C \\
\hline Ace & 24,98 & 26,27 & 17,44 & 28,51 \\
Ice & 25,85 & 30,26 & 18,04 & 28,28 \\
Chao 1 & $24,5 \pm 2,59$ & $24,2 \pm 1,84$ & $16,5 \pm 1,29$ & $27 \pm 1,81$ \\
Chao 2 & $24,1 \pm 1,71$ & $26,20 \pm 3,39$ & $16,91 \pm 1,68$ & $26,34 \pm 0,77$ \\
Jackknife 1 & $26,66 \pm 2,06$ & $29,41 \pm 2,12$ & $18,75 \pm 1,43$ & $28,75 \pm 1,43$ \\
Jackknife 2 & 26,96 & 31,46 & 19,73 & 25,95 \\
Bootstrap & 24,95 & 26,02 & 17,36 & 27,95 \\
\hline
\end{tabular}

*Ponto A- 500 m do portão da Reserva (borda); Ponto B- 1200 m do portão e distando 1000 m para o interior da mata; Ponto C - 1700 m do portão e distando $500 \mathrm{~m}$ para o interior da mata.

maiores valores (exceto Chao 1), provavelmente por possuir um maior número de espécies raras. Dias (2004) lembra que espécies raras são mais difíceis de serem capturadas por estarem em menor abundância que as comuns, portanto é possível que um maior tempo de procura (neste caso, exposição de armadilha) levasse ao encontro de um maior número de espécies.

A riqueza esperada da comunidade de califorídeos foi calculada por vários métodos que servem para saber o quão boa foi sua amostragem, uma vez que lhe mostra qual seria a riqueza esperada para uma determinada amostra com o intervalo de confiança. Amostras confiáveis apresentam um intervalo de confiança pequeno, e o valor de riqueza encontrado dentro desse intervalo. Assim, este trabalho apresentou apenas dois valores de riqueza estimada com intervalos de confiança grandes, o que segundo Vieira \& Mendel (2002) indica um bom esforço amostral e que o levantamento realizado se aproximou da realidade. Os estimadores se tornam mais precisos com o aumento do número de coletas (Colwell 2004).

Além disso, a utilização de vários estimadores pode provar qual deles é mais eficiente para cada ambiente (Palmer 1990). Nos pontos A e C, os estimadores Chao 1 e Chao 2 geraram valor menor que a riqueza observada considerando o desvio padrão. O mesmo ocorreu para Chao 1 no ponto B. Isso os torna pouco confiáveis. Os estimadores Ace e Bootstrap apresentaram-se mais seguros para estimativa de riqueza de espécies de Calliphoridae.

Em estudos divididos em amostras com grupos de animais que apresentam muitas espécies, como artrópodes, a diversidade tem como melhor opção de ser avaliada através dos estimadores de riqueza (Dias 2004). A riqueza de espécies descoberta é tão importante quanto o número de espécies a descobrir em propostas ecológicas conservacionistas (Santos 2003), sendo toda informação de riqueza e diversidade essencial para subsidiar políticas de conservação (Coddington et al. 1991). Portanto a Reserva Biológica do Tinguá apresenta uma grande riqueza de califorídeos, e avaliar outros grupos animais é uma boa alternativa para ressaltar ainda mais sua importância como área de preservação de espécies.

A Equidade J compara a diversidade de Shannon-Wiener com a distribuição das espécies observadas. Todas as amostras devem prover de um mesmo ambiente e deve conter amostragem suficiente para conter todas as espécies (Rodrigues 2004). As equidades nos pontos A, B e C foram baixas e bem semelhantes. A baixa eqüidade no ponto A reflete a alta dominância de C. albiceps e Hemilucilia semidiaphana (Rondani, 1850) na amostra, enquanto que no ponto B a dominância é dada pela $M$. bellardiana e no ponto C pela espécie L. nigripes. Estas espécies também estiveram presentes em áreas naturais em estudos de Tavares (2003), Thyssen (2000), Ribeiro (2003), exceto L. nigripes e, ainda, $M$. bellardiana apenas no último. Cabe ressaltar que $C$. albiceps trata-se de uma espécie introduzida no Brasil, porém vem mostrando-se bem adaptada. Da mesma forma, cita-se a espécie Chrysomya megacephala (Fabricius, 1794), cujo número de indivíduos capturados somados a de $C$. albiceps neste estudo, no ponto $\mathrm{A}$, somaram quase $50 \%$ do total capturado, no ponto $\mathrm{B}$, quase $10 \%$, e no ponto $\mathrm{C}$ próximo de $6 \%$.

A equidade, equitabilidade ou igualdade refere-se ao padrão de distribuição de indivíduos entre as espécies (Rodrigues 2004). Porém, a dominância é uma característica difícil de ser avaliada pois representa a ação de organismos dominantes sobre uma comunidade. Estes podem exercer sua ação sobre outros, causando seu aparecimento ou desaparecimento. Depende da atividade desempenhada pela espécie na comunidade (Silveira-Neto et al. 1976).

A diversidade de espécie refere-se à variedade de espécies de organismos vivos de uma determinada comunidade, habitat ou região (Rodrigues 2004). A diversidade pode dar maior estabilidade e atuar na sobrevivência da comunidade, já que até as espécies raras desempenham funções indiretas importantes. Os índices de diversidade são medidas características de cada comunidade, não dependendo do processo de levantamento e do tamanho da amostragem (Silveira-Neto et al. 1976). O índice de diversidade de ShannonWiener foi proposto por Shannon (1948), e combina riqueza com uniformidade. Ele atribui maior peso às espécies raras. É o melhor para ser usado em comparações caso não haja interesse em se separarem os dois componentes da diversidade. É relativamente independente do tamanho da amostra e apresenta uma distribuição normal, contanto que $\mathrm{N}$ seja um número inteiro. É apropriado para amostras aleatórias de espécies de uma comunidade ou sub-comunidade de interesse (Rodrigues 2004). Neste estudo, o ponto B, foi considerado mais diverso. Os índices de diversidade de califorídeos encontrados nos 
pontos do presente estudo foram superiores aos de Ribeiro (2003) na Reserva Biológica de Mogi Guaçu, São Paulo, cujos valores no verão foram considerados baixos. Já em Tavares (2003), na Reserva Florestal da Serra do Japi, Jundiaí, São Paulo, os índices de diversidade de califorídeos relativos ao verão e inverno de 1999 foram superiores aos encontrados no ponto C do nosso estudo e inferiores aos dos pontos A e B. No trabalho realizado por Thyssen (2000), na Reserva Municipal de Santa Genebra, Campinas, São Paulo, foi observado que a diversidade de califorídeos variou com o tamanho das carcaças utilizadas nas coletas.

Locais com fatores limitantes atuando intensamente e competição interespecífica, costumam apresentar baixos índices de diversidade, aumentam o número de espécies mais comuns e diminuem as raras, tornando o local mais específico. Em regiões tropicais, como em florestas, costuma ocorrer o contrário, pois abrigam diversos nichos, aumentando a diversidade, ocorrendo mais espécies com menos indivíduos (Silveira-Neto et al. 1976).

A similaridade obtida pelo índice de Sorensen entre os pontos $\mathrm{A}$ e B, A e C, e B e C foram todos acima de 0,5 , o que indica alta semelhança entre as parcelas (Santos \& Jardim 2006). Não podemos assim, neste quesito, caracterizar cada ponto como um micro habitat.

Os dendogramas foram similares. O primeiro dendograma agrupou os pontos $\mathrm{B}$ e $\mathrm{C}$ como mais próximos entre si, em relação ao ponto A. Da mesma forma, no segundo dendograma, as espécies L. nigripes e M. bellardiana aparecem separadas das demais, sendo elas as principais espécies dos pontos B e C. Estas espécies são assinantrópicas e portanto comuns em áreas florestadas (D’Almeida \& Lopes 1983). As espécies $C$. albiceps, C. megacephala e $H$. semidiaphana, principais espécies no ponto A, também aparecem unidas, em outro agrupamento.

Comparações com estudos anteriores na Reserva Biológica do Tinguá podem trazer maiores esclarecimentos e manter a informação da preservação das espécies, assim como a continuação destes trabalhos, já que a abundância de muitas espécies de insetos tropicais muda sazonalmente (Wolda 1980) e também por ação antrópica nessas áreas. Este estudo pode auxiliar ainda nas investigações forenses a respeito do local de ocorrência do crime, pela demonstração de diferentes padrões de ocupação de espécies em pontos próximos em um mesmo fragmento de mata.

Agradecimentos. À Margareth M. C. Queiroz e a Fundação Oswaldo Cruz/RJ pelo veículo utilizado nas coletas. À CAPES, CNPq e UNIRIO pelo apoio financeiro.

\section{REFERÊNCIAS}

Bragagnolo, C. \& R. Pinto-da-Rocha. 2003. Diversidade de Opiliões do Parque Nacional da Serra dos Órgãos, Rio de Janeiro, Brasil (Arachnida: Opiliones). Biota Neotropica 3: 1-13.

Chao, A. 1984. Non-parametric estimation of the number of classes in a population. Scandinavian Journal of Statistics 11: 265-270.

Chao, A. 1987. Estimating the Population Size for Capture-
Recapture Data with Unequal Catchability. Biometrics 43: 783-791

Coddington, J. A.; C. E. Griswold; D. Silva Dávila; E. Peñaranda \& S. F. Larcher. 1991. Designing and testing sampling protocols to estimate biodiversity in tropical ecosystems, p. 44-60 In: Dudley, E. C. (ed.) The unity of evolutionary biology: proceedings of the fourth International Congress of Systematic and Evolutionary Biology. Portland, Dioscorides Press, $231 \mathrm{p}$.

Colwell, R. K. 2004. User's guide to EstimateS5 statistical. Estimation of species richness and shared species from samples. Version 7.0.0.Copyright 1994-2004. Disponível em http://viceroy.eeb.uconn.edu/estimates. Acessado em: 24/10/2004.

Colwell, R. K. 2006. Estimates: estatistical estimation of species richness and shared species from samples. Version 8.0. Disponível em: http://viceroy.eeb.uconn.edu/estimate. Acessado em: 25/08/2006.

Colwell, R. K. \& J. A. Coddington. 1994. Estimating terrestrial biodiversity through extrapolation. Philosophical Transactions Royal Society of London 345: 101-118

D’Almeida, J. M. \& H. S. Lopes.1983. Sinantropia de Dípteros Muscóides (Calliphoridae) no Estado do Rio de Janeiro. Arquivo da Universidade Federal Rural do Rio de Janeiro 6: 39-48.

De Souza, O.; J. H. Schoereder; V. Brown \& R. O. Bierregaard Jr. 2001. A theoretical overview of the process determining species richness in forest fragments, p. 13-21. In: R. O. Bierregaard Jr.; C. Gascon; T. E. Lovejoy \& R. C. G. Mesquita (eds.). Lessons from Amazonia: the ecology and conservation of a fragmented forest. New Haven, USA, Yale University Press, 478 p.

Dias, S. C. 2004. Planejando estudos de diversidade e riqueza: uma abordagem para estudantes de graduação. Acta Scientiarum Biological Sciences 26: 373-379.

Erwin, T. L. 1988. The tropical forest canopy-The heart of biotic diversity. p 123-129 In: E.O. Wilson (ed.). Biodiversity. Washington, National Academy Press, 538 p.

Ferraz, A. C. P. \& V. M. Aguiar-Coelho. 2008. Desenvolvimento e Avaliação de Novas Metodologias para Testar a Atratividade de Chrysomya megacephala (Fabricius) (Diptera: Calliphoridae) a Estímulo Visual por Cores em Condições Laboratoriais. Neotropical Entomology 37: 334-337.

Furusawa, G. P. \& P. C. R. Cassino. 2006. Ocorrência e distribuição de Calliphotidae (Diptera: Oestroidea) em um Fragmento de Mata Secundária no Município de Paulo de Frontin, Médio Paraíba, RJ. Revista de Biologia e Ciências da Terra 6: 152-164.

Greenberg, B. 1973. Flies and disease: biology and disease transmission. New Jersey, Princeton University, 447 p.

Guimarães J. H.; N. A. Papavero \& A. P. Prado. 1983. As miíases na região Neotropical (identificação, biologia e bibliografia). Revista Brasileira de Zoologia 1: 293-416.

Holloway, J. D.; J. D. Bradley \& J. D. Carter. 1987.Guides to insects of importance to man. Lepidoptera, 1. Wallinford, C.A.B. International., $62 \mathrm{p}$.

Huston, A. H. 1995. Biological diversity: the coexistence of species on changing landscapes. Cambridge, Cambridge University, 681p.

Hutcheson, K. 1970. A test for comparing diversities based on the Shannon formula. Journal of Theoretical Biology 29: 151154.

Ibama. 2006. Unidade: Reserva Biológica do Tinguá. Disponível em: http://www.ibama.gov.br/siucweb/mostraUc.php?seqUc $=42$. Acessado em 20/04/06.

Kruger, R. F. 2006. Analise da riqueza e da estrutura das assembléias de Muscidae (Diptera) no bioma Campos Sulinos, Rio Grande do Sul, Brasil. Tese de doutorado, Universidade Federal do Paraná. Disponível em: http:// dspace.c3sl.ufpr.br/dspace/bitstream/1884/3833/1/Tese.pdf

Lee, S. M. \& A. Chao. 1994. Estimating population size via sample coverage for closed capture-recapture models. Biometrics 50: 88-97.

Longino, J. T.; J. Coddington \& R. K. Colwell. 2002. The ant fauna of a tropical rain forest: estimating species richness in three different ways. Ecology 83: 689-702 
Magurran, A. E. 1988. Ecological diversity and its measurement. New Jersey, Princeton University Press, 179 p.

Margalef, R. 1951. Diversidad de especies en las comunidades naturales. Publicaciones del Instituto de Biologia Aplicada e Barcelona 9: $5-27$.

Mello, R. S.; M. M. C. Queiroz \& V. M. Aguiar-Coelho. 2007. Population fluctuations of calliphorid species (Diptera, Calliphoridae) in the Biological Reserve of Tinguá, state of Rio de Janeiro, Brazil. Iheringia Série Zoologia 97: 1-5.

Mello, R. P. 2003. Chave para a identificação das formas adultas das espécies da família Calliphoridae (Diptera, Brachycera, Cyclorrhapha) encontradas no Brasil. Entomologia y Vectores 10: $255-268$.

Moreno, C. E. 2001. Métodos para medir la biodiversidad. vol.1. Zaragoza, M\&T-Manuales y Tesis SEA, 84 p.

Moreno, C. E. \& G. Halffter. 2001. Spatial and temporal analysis of a, $\mathrm{b}$ and $\mathrm{g}$ diversities of bats in a fragmented landscape. Biodiversity and Conservation 10: $367-382$.

Moura, M. O.; C. J. B. Carvalho; E. L. A. Monteiro-Filho. 1997. A preliminary analysis of insects of medico-legal importance in Curitiba, States of Paraná. Memórias do Instituto Oswaldo Cruz, 92: 269-274.

Neves, D. P. 2005. Parasitologia Humana. São Paulo, Editora Atheneu, $494 \mathrm{p}$.

Palmer, M. W. 1990. The estimation of species richness by extrapolation. Ecology 71: 1195-1198.

Palmer, M. W. 1991. Estimating species richness: The second-order jackknife reconsidered. Ecology 72: 1512-1513.

Pielou, E. C. 1975. Ecological diversity. John Wiley \& Sons, Inc., New York, 165 pp.

Pielou, E. C. 1966. An introduction to mathematical ecology. New York, John Wiley \& Sons. 286 p.

Primack, R. B. \& E. Rodrigues. 2001. Biologia da conservação. Londrina, E. Rodrigues. 328 p.

Ribeiro, N. M. D. 2003. Decomposição e sucessão entomológica em carcaças de suíno expostas em área de cerrado e mata ciliar do Sudeste Brasileiro. Tese Mestrado em Parasitologia. Universidade Estadual de Campinas, SP. 69p. Disponível em: http:/ /libdigi.unicamp.br/document/?code $=$ vtls000300671

Rodrigues, W. C. 2004. Homópteros (Homoptera: Sternorrhyncha) associados à tangerina cv. Poncã (Citrus reticulata Blanco) em cultivo orgânico e a interação com predadores e formigas. Tese Doutorado em Fitotecnia. Universidade Federal Rural do Rio de Janeiro. Seropédica. $63 \mathrm{p}$.

Santos, A. J. 2003. Estimativas de Riqueza em Espécies. In Métodos de Estudos em Biologia da Conservação e Manejo da Vida Silvestre (L. Cullen Jr., R. Rudran \& C. Valladares-Pádua, orgs.). Curitiba, Editora da Universidade Federal do Paraná.

Santos, G. C. \& M. A. G. Jardim. 2006. Floristic and structure of tree communities in the floodplain forest on municipallity of Santa Bárbara do Pará, State of Pará, Brazil. Acta Amazonica 36: $437-$ 446.

Shannon, C. E. 1948. A mathematical theory of communication. Bell System Technical Journal 27: 379-423.

Silva, L. P. 2001. Um pouco sobre a Reserva Biológica do Tinguá. Disponível em: http://inema.com.br/mat/idmat001103.htm. Acessado em: 23/04/06

Silva, M. S.; J. C. Fontenelle \& R. P. Martins. 2001. Por que moscas visitam flores? Ciência Hoje 30: 68-71.

Silveira-Neto, S.; O. Nakano; D. Barbin \& N. A. V. Nova. 1976. Manual de ecologia de insetos. São Paulo, Ed. Agronômica Ceres. 419p.

Smith, E. P. \& G. Van Belle.1984. Nonparametric estimation of species richness. Biometrics 40: 119-129.

Sorensen, T. 1948. A method of establishing group of equal amplitude in plant sociology based on similarity of species content and its application to analyses of the vegetation on Danish commons. Biologiske Skrifter 5: 1-34.

Southwood, T. R. E. 1978. Ecological methods. Chapman \& Hall, New York.

Southwood, T. R. F. 1995. Ecological methods. 2nd. ed., London, Chapman \& Hall. 524 p.

Tavares, M. C. H. 2003. Sucessão faunística de populações de insetos associados à decomposição de carcaças de suínos expostas em diferentes altitudes e condições pluviométricas na Reserva Florestal da Serra do Japi, Jundiaí, SP.Tese Doutorado em Parasitologia. Universidade Estadual de Campinas, $111 \mathrm{p}$.

Thyssen, P. J. 2000. Decomposição e sucessão entomologica em carcaças de suinos (Sus scrofa L.) de tamanhos diferentes : estudos em ambiente de mata natural na região de Campinas - SP. Tese Mestrado em Parasitologia. Universidade Estadual de Campinas, SP. 75p. Disponível em: http://libdigi.unicamp.br/ document $/$ ?code $=$ vtls 000197613

Toti, D. S.; F. A. Coyle \& J. A. Miller. 2000. A structured inventory of Appalachian grass bald and heath bald spider asemlages and a test of species richness estimator performance. Journal of Arachnology 28: 329-345.

Vieira, L. M. \& S. M. Mendel. 2002. Riqueza de artrópodes relacionada à complexidade estrutural da vegetação: uma comparação entre métodos. In: Ecologia de Campo - Curso de Campo 2002. Campo Grande, Venticique E. \& Hopkins M.

Whittaker, R. H. 1967. Gradient analysis of vegetation. Biological Reviews Cambridge 42: 207-264.

Wolda, H. 1980. Seasonality of tropical insects. I. Leafhoppers (Homoptera) in Las Cumbres, Panama. Journal of Animal Ecology 49: 277-290.

Zar, J. H. 1999. Biostatitical Analysis. 3. ed. New Jersey, Prentice Hall.

Recebido em 08/05/2008; aceito em 15/10/2009 